\title{
Asiago eclipsing binaries program IV. SZ Camelopardalis, a $\beta$ Cephei pulsator in a quadruple, eclipsing system ${ }^{\star}$
}

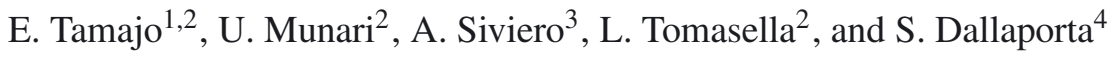 \\ ${ }^{1}$ Department of Physics, University of Zagreb, Bijenička cesta 32, 10000 Zagreb, Croatia \\ e-mail: etamajo@phy.hr \\ 2 INAF Astronomical Observatory of Padova, 36012 Asiago (VI), Italy \\ 3 Department of Astronomy, University of Padova, 35122 Padova (PD), Italy \\ 4 ANS Collaboration, c/o Osservatorio Astronomico, via dell'Osservatorio 8, 36012 Asiago (VI), Italy
}

Received 5 August 2011 / Accepted 16 January 2012

\section{ABSTRACT}

\begin{abstract}
We present a spectroscopic and photometric analysis of the multiple system and early-type eclipsing binary SZ Cam (O9 IV + B0.5 V), which consists of an eclipsing SB2 pair of orbital period $P=2.7$ days in a long orbit ( $~ 55$ yrs) around a non-eclipsing SB1 pair of orbital period $P=2.8$ days. We have reconstructed the spectra of the individual components of SZCam from the observed composite spectra using the technique of spectral disentangling. We used them together with extensive and accurate $B V I_{\mathrm{C}} \mathrm{CCD}$ photometry to obtain an orbital solution. Our photometry revealed the presence of a $\beta$ Cep variable in the SZCam hierarchical system, probably located within the non-eclipsing SB1 pair. The pulsation period is $(0.33265 \pm 0.00005)$ days and the observed total amplitude in the $B$ band is $(0.0105 \pm 0.0005)$ mag. NLTE analysis of the disentangled spectra provided atmospheric parameters for all three components, consistent with those derived from orbital solution.
\end{abstract}

Key words. binaries: close - binaries: eclipsing - stars: fundamental parameters

\section{Introduction}

In the past several decades, new theoretical models have been established in order to include a number of physical processes and phenomena, which are important for understanding the structure and evolution of high-mass stars. These processes are convective core-overshooting, semi-convection, rotational material mixing and mass loss due to stellar winds. However, empirical constraints on these features remain of high priority, despite steady improvement in observational techniques and capabilities (Hilditch 2004). Eclipsing binaries of the detached type are a great resource for obtaining accurate values of stellar masses, radii, and luminosities (Andersen 1991; Torres et al. 2010). Given that the component stars have the same age and initial chemical composition, eclipsing binaries have been extensively used to test predictions of stellar models (e.g. Siviero et al. 2004; Tomasella et al. 2008aa,b).

SZ Cam (=HD $25638 \mathrm{~V}=6.9 \mathrm{mag}$ ) is an early type eclipsing binary (B0 II-III + O9.5 V according to Morgan et al. 1955; Budding 1975), which is a member of the very young open cluster NGC 1502. For this cluster, the compilation of literature data by Dias et al. (2002) lists an age of $10 \mathrm{Myr}$, a distance of $1.0 \mathrm{kpc}$, and a radial velocity of $-9.7 \mathrm{~km} \mathrm{~s}^{-1}$. The photometric variability of SZCam was discovered by (Guthnick \& Prager 1930), and light curves were presented by Wesselink (1941), Olsen (1961), Kitamura \& Yamasaki (1971), Polushina (1977), Chochol (1980), Gorda \& Polushina (1987), and Gorda (2000).

* Full Table 3 is only available at the CDS via anonymous ftp to cdsarc.u-strasbg.fr $(130.79 .128 .5)$ or via http://cdsarc.u-strasbg.fr/viz-bin/qcat?]/A+A/539/A139
Their observations were carried out by means of photographic or photoelectric photometry. The photoelectric observations can attain a high intrinsic accuracy, but not in the case of open clusters where many other field stars enter the aperture of the photometer (usually several tens of arcsec wide), and even less so in the case of SZ Cam that has a similarly bright star (HD 25639), itself member of NGC 1502 and a variable star, just 18 arcsec away. All light curves of SZ Cam so far published have been judged by these authors to be noisier than expected from the high brightness of the target star. This was attributed to the perturbation of the photometric measure by nearby stars, and no investigation of an intrinsic variability has been carried out in addition to the eclipse modulation.

SZ Cam was first recognized as a spectroscopic binary by Plaskett (1924), and spectroscopically was found to be a triple stellar system by Mayer et al. (1994, 2010). The presence of the third component has been confirmed by speckle interferometry (Mason et al. 1998). More speckle measurements were published by Gorda et al. (2007) and Balega et al. (2007). These speckle measurements detected an orbital motion for the tertiary component but one that is extended long enough in time to cover only a small arc of the overall orbit. Lorenz et al. (1998) and Michalska et al. (2007) have found that the tertiary star is itself a close, single-lined binary, thus making SZ Cam a quadruple system.

The main parameters of the orbital solutions so far published (Harries et al. 1998; Lorenz et al. 1998; Michalska et al. 2007; Gorda 2008; Mayer et al. 2010) are based on radial velocities obtained over limited wavelength ranges (sometimes just a single line, as in the case of $\mathrm{H} \alpha$ for Michalska et al. 2007), sometimes on low signal-to-noise ratio $(\mathrm{S} / \mathrm{N})$ spectra or at medium to low 
Table 1. Comparison of various orbital solutions for the eclipsing pair in the quadruple system SZCam.

\begin{tabular}{|c|c|c|c|c|c|c|}
\hline & Harries et al.(1998) & Lorenz et al. (1998) & Michalska et al. (2007) & Gorda (2008) & Mayer et al. (2010) & this paper \\
\hline$K_{1}\left(\mathrm{~km} \mathrm{~s}^{-1}\right)$ & $225.8 \pm 3.8$ & $180.2 \pm 2.0$ & 181.6 & $192.0 \pm 2.6$ & $189.4 \pm 1.4$ & $185.2 \pm 2.8$ \\
\hline$K_{2}\left(\mathrm{~km} \mathrm{~s}^{-1}\right)$ & $259.1 \pm 4.0$ & $261.2 \pm 3.8$ & 268.2 & $266.4 \pm 2.5$ & $264.1 \pm 2.5$ & $247.0 \pm 3.6$ \\
\hline$M_{1}\left(M_{\odot}\right)$ & $18.5 \pm 0.7$ & $15.26 \pm 0.53$ & & $16.65 \pm 0.38$ & $16.6 \pm 0.4$ & $14.31 \pm 0.54$ \\
\hline$M_{2}\left(M_{\odot}\right)$ & $16.1 \pm 0.6$ & $10.75 \pm 0.44$ & & $12.01 \pm 0.33$ & $11.9 \pm 0.3$ & $10.69 \pm 0.38$ \\
\hline$q=M_{2} / M_{1}$ & $0.871 \pm 0.026$ & $0.690 \pm 0.015$ & 0.68 & $0.72 \pm 0.02$ & $0.717 \pm 0.035$ & $0.747 \pm 0.006$ \\
\hline$R_{1}\left(R_{\odot}\right)$ & $8.5 \pm 0.3$ & $9.01 \pm 0.91$ & & & $9.4 \pm 0.2$ & $8.91 \pm 0.05$ \\
\hline$R_{2}\left(R_{\odot}\right)$ & $7.9 \pm 0.3$ & $6.63 \pm 0.68$ & & & $5.4 \pm 0.2$ & $6.70 \pm 0.12$ \\
\hline$T_{1}(K)$ & $29725 \pm 500$ & 33000 & & & 30500 & $30360 *$ \\
\hline$\Delta T_{2}(K)$ & $2542 \pm 724$ & $4950 \pm 500$ & & & $5200 \pm 740$ & $3116 \pm 255$ \\
\hline$\gamma\left(\mathrm{km} \mathrm{s}^{-1}\right)$ & $-12.0 \pm 3.0$ & $-2.9 \pm 1.6$ & & $-10.6 \pm 2.0$ & -2.3 & \\
\hline$l_{3}{ }^{* *}$ & $27.4 \%$ & $20 \%$ & & & $30 \%$ & $25.5 \pm 0.7 \%$ \\
\hline$e$ & 0 & 0 & & & 0 & 0 \\
\hline
\end{tabular}

Notes. ${ }^{(*)}$ Assumed from NLTE modeling not constrained by orbital solution (see Table 4$){ }^{(* *)}$ The value of $l_{3}$ is for the $V$ photometric band.

resolving powers. The techniques used to measure the radial velocities varied from multipeaked cross-correlation functions, to simple multi-Gaussian fitting to some individual lines, to spectral disentangling of a single line $(\mathrm{H} \alpha)$. The resulting orbital parameters differ from one published solution to the other, much more than the quoted errors as illustrated in Table 1.

The motivation for this new effort on SZ Cam is multifold: ( $i)$ to obtain a complete new set of high accuracy $B V I_{\mathrm{C}}$ photometry with long- focus CCD imaging, able to accurately split SZ Cam from any surrounding disturbing field star and thus greatly improving on existing photometric data. In addition to providing better eclipse light curves, these data are used to investigate and characterize any intrinsic variability displayed by any of the four components. We have indeed discovered that one of them is a $\beta$ Cep pulsator; (ii) to derive the radial velocities of the components of SZ Cam via spectral disentangling of the whole optical spectrum from REOSC Échelle high-resolution data. Without limiting the RV measurement to just a few lines of assumed Gaussian shape as happened in previous studies, we are confident the results should gain in accuracy; (iii) to derive with NLTE analysis the temperature and gravity of the stellar components of SZ Cam, and compare them with the results of orbital solution and with expectation from theoretical isochrones.

\section{Observations}

\subsection{Spectroscopy}

The spectra of SZ Cam were obtained in 2005 and 2011 with the REOSC Échelle + CCD spectrograph on the $1.82 \mathrm{~m}$ telescope operated by Osservatorio Astronomico di Padova at Mt. Ekar (Asiago). The observing log is given in Table 2. A 2-arcsec slit was adopted with fixed E-W orientation, producing a PSF with an FWHM of 1.75 pixel over the whole observing campaign, corresponding to a resolving power close to $R_{\mathrm{p}} \sim 20000$. The PSF is measured on the night sky and on comparison lamp emission lines (and confirmed by analysis of telluric $\mathrm{O}_{2}$ and $\mathrm{H}_{2} \mathrm{O}$ absorption lines). Even if uniformly illuminating the slit, the night sky and comparison lamp emission lines are considered a fair approximation of the stellar illumination mode of the slit given the typical seeing (around 2 arcsec) and the deliberate manual guiding of the star on the slit for half an hour each exposure. The manual guiding was preferred over the equally possible automatic one to avoid introducing spurious, low-amplitude velocity shifts caused by the star not being kept exactly on the slit center by the auto-guiding.
Table 2. Journal of observations.

\begin{tabular}{|c|c|c|c|c|c|c|c|}
\hline ID & $\begin{array}{c}\text { HJD } \\
(-2450000)\end{array}$ & Phase & $\mathrm{S} / \mathrm{N}$ & ID & $\begin{array}{c}\text { HJD } \\
(-2450000)\end{array}$ & Phase & $\mathrm{S} / \mathrm{N}$ \\
\hline 40964 & 3314.479 & 0.0511 & 128 & 41729 & 3425.479 & 0.9154 & \\
\hline & & & 144 & & & & \\
\hline & & & & & & & 2 \\
\hline & & & 38 & & 0 & .9076 & 6 \\
\hline 219 & 7 & & 140 & 34 & 05 & 0.90 & 9 \\
\hline & 75 & 4052 & 129 & 36 & 3425 . & 0.89 & 8 \\
\hline & & 608 & 127 & & 26 & 0.8980 & 5 \\
\hline & & & 130 & & & & 52 \\
\hline & & & 00 & & & & 5 \\
\hline & & & 107 & & & & 3 \\
\hline & & & 91 & & & & 32 \\
\hline & & & 171 & & & & 43 \\
\hline & & & 169 & & & & 34 \\
\hline & & & 97 & & & & 43 \\
\hline & & & 125 & & & & 80 \\
\hline & & & 29 & & & & \\
\hline & & & 47 & & & & 107 \\
\hline & & & 40 & & & & 91 \\
\hline & & & 30 & & & & 171 \\
\hline 41728 & & 0.9172 & 62 & & 5663.272 & 0.3930 & 169 \\
\hline
\end{tabular}

Notes. The $\mathrm{S} / \mathrm{N}$ is computed on the continuum at $5870 \AA$. The timescale is UTC.

Table 3. $B V I_{\mathrm{C}}$ photometric data for SZCam. HJD = heliocentric JD - 2455000 .

\begin{tabular}{|c|c|c|c|c|c|c|}
\hline Date & HJD & err & $\overline{B-V}$ & err & $\overline{V-I_{\mathrm{C}}}$ & err \\
\hline 20110211.120 & 603.620 & 6.9380 .008 & 0.421 & 0.007 & 0.527 & 0.004 \\
\hline 20110211.111 & 603.611 & 6.9500 .005 & 0.408 & 0.006 & 0.536 & 0.004 \\
\hline 20110211.102 & 603.602 & 6.9410 .006 & 0.423 & 0.006 & 0.540 & 0.004 \\
\hline 20110211.090 & 603.590 & 6.9390 .005 & 0.431 & 0.005 & 0.523 & 0.006 \\
\hline 20110211.078 & 603.578 & 6.9350 .007 & 0.437 & 0.009 & 0.529 & 0.007 \\
\hline 20110211.074 & 603.574 & 6.9260 .006 & 0.438 & 0.007 & 0.512 & 0.004 \\
\hline 20110211.070 & 603.570 & 6.9430 .006 & 0.419 & 0.006 & 0.542 & 0.004 \\
\hline 20110211.066 & 603.566 & 6.9380 .006 & 0.419 & 0.006 & 0.533 & 0.002 \\
\hline
\end{tabular}

Notes. The timescale is UTC. Full Table 3 is available at the CDS. A portion is shown here for guidance regarding its form and content.

Exposures of a thorium lamp for wavelength calibration were obtained both immediately before and soon after the exposures on SZCam, on which the telescope was still tracking. These two exposures of the thorium lamp were combined before 
E. Tamajo et al.: Asiago eclipsing binaries program. IV.

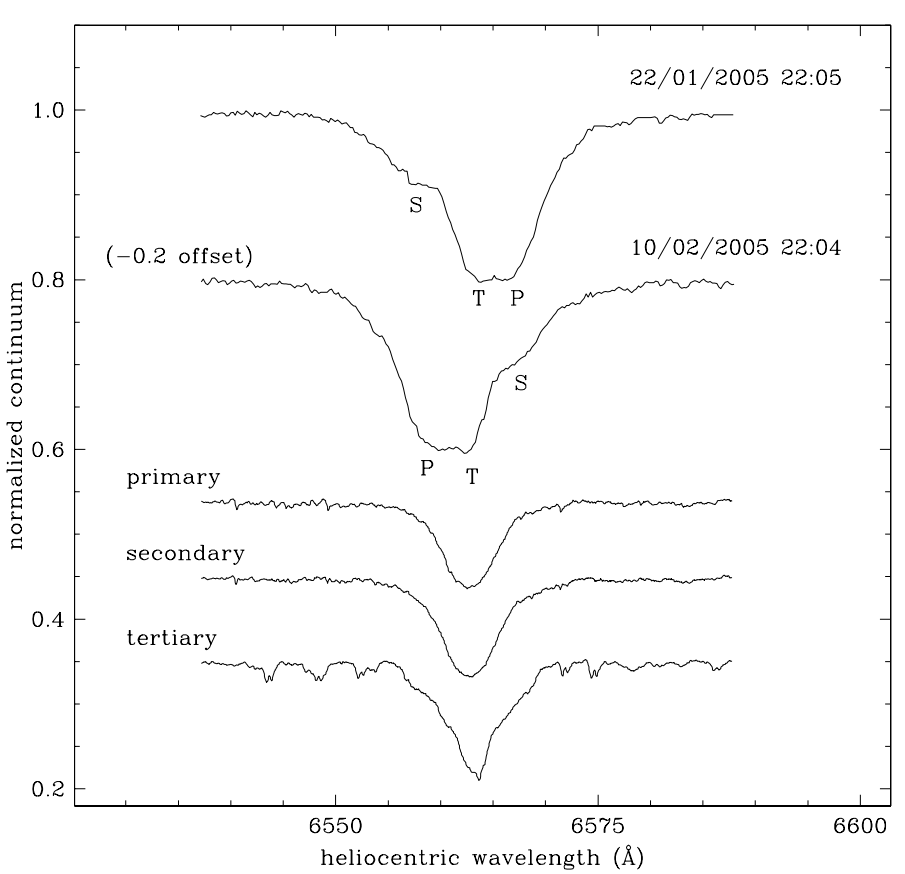

Fig. 1. Two examples of $\mathrm{H}_{\alpha}$ profiles for SZCam from Asiago REOSC Échelle observations. The features of the primary (P), secondary (S) and tertiary (T) stars are labeled. The three lower curves are the spectra of the three individual components, normalized to their light contribution, as we disentangled from the whole set of 40 REOSC Échelle spectra covering all orbital phases.

extraction, to compensate for spectrograph flexures. From the start of the first thorium exposure to the end of the last, the whole observing cycle including the exposure on SZ Cam took about 40 min. According to the detailed investigation and 2D modeling by Munari \& Lattanzi (1992) of the flexure pattern of the REOSC Échelle spectrograph mounted at the Asiago $1.82 \mathrm{~m}$ telescope, and considering that we imarily observed our target when it was crossing the meridian, the impact of spectrograph residual flexures on our observations corresponds to an uncertainty below $0.2 \mathrm{~km} \mathrm{~s}^{-1}$, thus completely negligible in the context of our study. The absence of a systematic velocity off-set and of random velocity errors lower than $0.3 \mathrm{~km} \mathrm{~s}^{-1}$ are confirmed by $(i)$ the measurement by cross-correlation of the radial velocity of the rich telluric absorption spectrum in the red portion of all our spectra, and (ii) the measurement of all night sky lines we detected in our spectra, relative to the compilations of their wavelengths by Meinel et al. (1968), Osterbrock \& Martel (1992), and Osterbrock et al. (1996).

\section{2. $C C D$ photometry}

CCD photometry on SZCam was collected in Johnson's $B$ and $V$ bands and Cousin's $I_{C}$. A total of $1517 B V I_{C}$ observing sequences were obtained in 43 different observing nights, from August 28, 2010 to February 11, 2011. They are reported in Table 3. Three minima were covered well by continuous monitoring throughout the descending, minimum, and ascending branches. The time of central eclipse for them occurred at HJD 462.5310, 535.3861, and 562.3749 (+2 455000$)$.

The telescope was a $0.3 \mathrm{~m} \mathrm{f} / 8$ Marcon Richey-Chretien telescope, privately owned by one of us (S.D.) and operated in Cembra (Trento, Italy). It was equipped with an SBIG ST-8 CCD camera, $1530 \times 1020$ array, $9 \mu$ m pixels $\equiv 0.77^{\prime \prime} /$ pix, with a field of view of $19^{\prime} \times 13^{\prime}$. The $B V I_{\mathrm{C}}$ filters are from Schuler.

The observations were treated in the usual fashion for bias, dark, and flat-field frames collected in each of the nights when SZCam was observed. The photometric measurements were performed with aperture photometry, with optimally chosen values for radius of aperture and internal and external radii of sky annulus. The optimal values were automatically and iteratively set by the condition of minimizing the rms of the many local photometric standard stars on each observation (see next paragraph) from the linear color equations. In spite of being located within the well-populated open cluster NGC 1502, the accuracy of aperture photometry was not affected by the presence of nearby cluster members, as extensive tests with PSF fitting photometry proved. The long focal length, good seeing, accurate auto-guiding, and the excellent optical quality of the RicheyChretien telescope were all instrumental to achieve this result.

The zero point and color equations of the photometry were calibrated against the $B, V$ photometric sequence of Hoag (1961) for NGC 1502 and against the $V-I_{C}$ of the comparison sequence calibrated by Henden \& Munari (2006) around the nearby CI Cam. The median slope of the $V$ color equation was 0.009 with a semi-interquartile range of 0.008 . The median and the semi-interquartile ranges were 1.008 and 0.017 for the $B-V$ color equation, with 0.993 and 0.013 for $V-I_{C}$. The errors reported in Table 3 are the total budget errors, which are the quadratic sum of the Poissonian error and the error associated to the transformation to the standard system as defined by the Hoag (1961) and Henden \& Munari (2006) standard stars. The median and semi-interquartile ranges of the total error budgets for $V, B-V$, and $V-I_{\mathrm{C}}$ are 0.006 and $0.001,0.007$ and 0.002 , 0.004 and $0.001 \mathrm{mag}$, respectively.

\section{Spectral disentangling}

\subsection{The method}

The technique of spectral disentangling (hereafter SPD) allows isolation of the spectra of the individual component stars of a double-lined spectroscopic binary system, using as input many observed spectra distributed well in orbital phases. It was originally formulated in the wavelength domain by Simon \& Sturm (1994) and in the Fourier domain by Hadrava (1995). The technique simultaneously returns the best-fitting spectra and the orbital velocity amplitudes of the two stars making up the binary. A detailed overview of SPD can be found in Pavlovski \& Hensberge (2010). The disentangled spectrum for each component of the binary contains the combined signal of all the input spectra and has a much higher $\mathrm{S} / \mathrm{N}$ compared to them. The total $\mathrm{S} / \mathrm{N}$ value in a disentangled spectrum of component $n$ is $S_{\text {dis }} \sim f_{n} S_{\text {obs }} \sqrt{N_{\text {obs }}}$, where $f_{n}$ is the fractional light contribution for component $n$ and $S_{\text {obs }}$ and $N_{\text {obs }}$ are the $\mathrm{S} / \mathrm{N}$ and number of input spectra.

The spectra of the individual components can be analyzed in the same way as those of single stars (Hensberge \& Pavlovski 2007; Pavlovski 2004; Pavlovski \& Southworth 2009; Pavlovski et al. 2009; Pavlovski \& Hensberge 2005). The usual degeneracy between $T_{\text {eff }}$ and $\log g$ which are so common in the atmospheric investigation of isolated single stars, is lifted in the case of eclipsing binaries by the very precise $\log g$ provided directly by the orbital solution (Hensberge et al. 2000; Simon et al. 1994).

Compared to other methods of radial velocity measurement, SPD has some advantages. First of all it is independent of template spectra, so it avoids any systematic errors due to spectral 

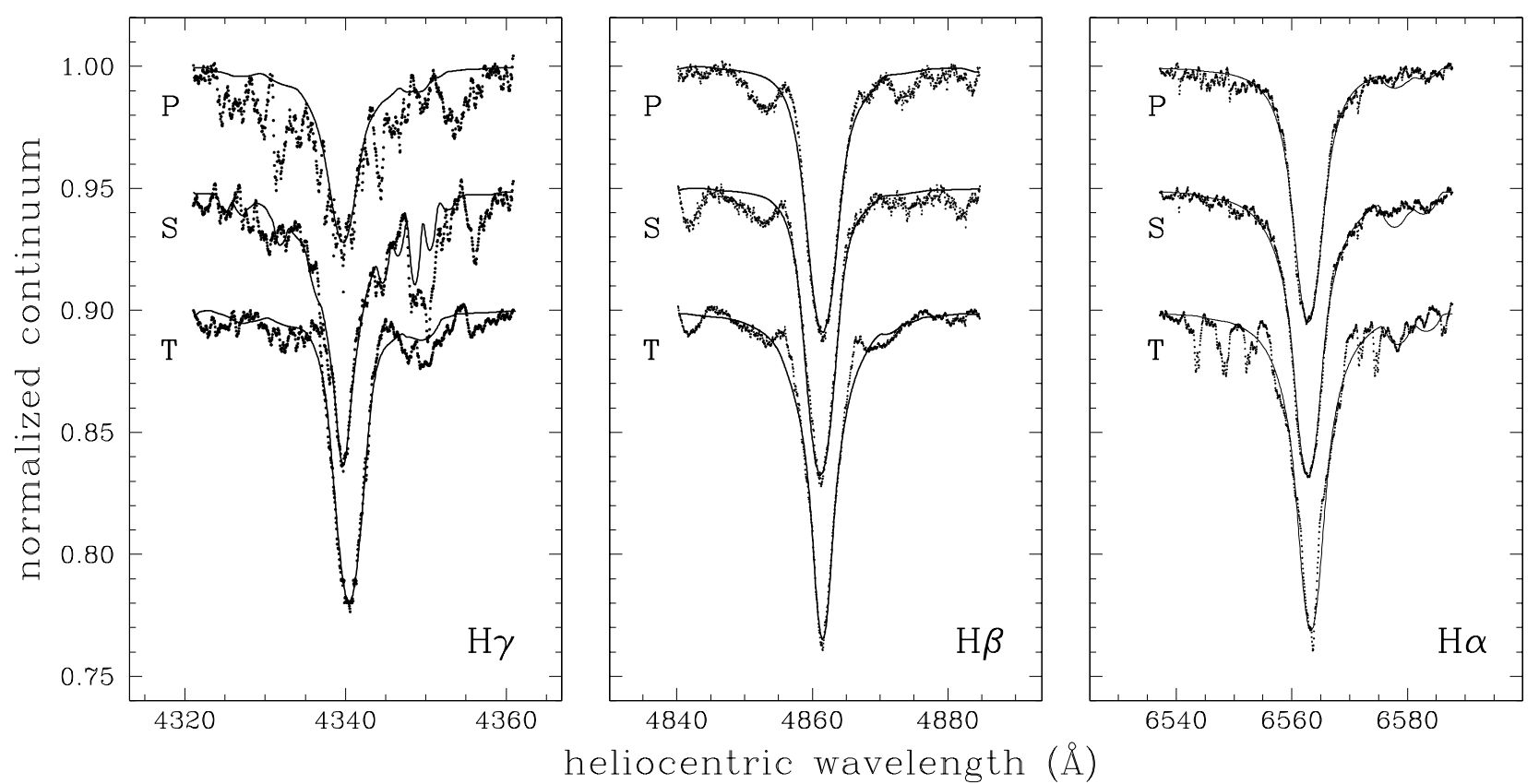

Fig. 2. Comparison between the disentangled spectra (points) and best-fitting NLTE theoretical spectra (solid lines) for $\mathrm{H}_{\gamma}, \mathrm{H}_{\beta}$ and $\mathrm{H}_{\alpha}$ Balmer-line. Labels P, S, and T mark the primary, secondary, and tertiary components, respectively.

differences between the target and template stars. Second, disentangling is not affected by the blending of spectral lines of the two stars (see Hensberge et al. 2000; Southworth \& Clausen 2007). Third, it does not require the real lines to resemble specific profiles (Gaussians) or to be symmetric. These two conditions are usually implicitly assumed in many investigations where blended line profiles are fitted with combinations of multiple Gaussians.

There are also a few disadvantages of the SPD approach. The first of these is that the continuum normalization of the input spectra has to be very precise in order to avoid lowfrequency spurious patterns in the resulting disentangled spectra (Hensberge et al. 2008). Another disadvantage is that relative continuum light contributions of the two stars cannot be found using SPD, since this information is itself not contained in the observed spectra, unless a spectrum has been obtained during an eclipse (Ilijić et al. 2004).

The SPD can equally treat eccentric and circular orbits. SZ Cam has been proven by previous studies (and confirmed by our photometric solution below) to be characterized by zero eccentricity. To check it further, we first run the SPD by letting unconstrained the eccentricity, and got no significant deviation from $e=0$. Consequently, we fixed a zero eccentricity and ran a final SPD that provided the results used in this paper. In setting $e=0$, the SPD converges on the spectra of the individual components that provide the best fit to a sinusoidal radial velocity variation. Therefore, its output amplitude is due to the orbital velocity variation (and its error).

To characterize the component stars of the binary, we used the method of fitting synthetic NLTE spectra to the disentangled ones, where the atmospheric parameters and the projected rotational velocities are determined simultaneously with the relative light contributions of the stars. A genetic algorithm (GENFITT, Tamajo 2009) is used for the optimization (Tamajo et al. 2011) in order to ensure that the best solution is found in a parameter space that suffers from strong degeneracies, in particular between effective temperature $\left(T_{\text {eff }}\right)$ and surface gravity $(\log g)$.
Table 4 compares the results of the NLTE analysis when it is run un-constrained and when the gravities of the two stars member of the eclipsing pair are fixed to the values derived by the orbital solution of Table 5. The rotational velocities of the tertiary stars are corrected for the smearing introduced by its orbital motion around an unseen companion.

\subsection{Application to SZCam}

We analyzed the Asiago REOSC Échelle spectra of SZ Cam obtained at Mt.Ekar about six years apart, using the SPD procedure analysis as implemented in the FDBINARY code (Ilijić et al. 2004), including all the 40 observed spectra in Table 2. We weighted the input spectra according to their $\mathrm{S} / \mathrm{N}$ value.

The procedure of SPD was performed in several spectral regions. We converged to accurate results for the regions of the prominent hydrogen Balmer lines $\mathrm{H}_{\alpha}, \mathrm{H}_{\beta}$, and $\mathrm{H}_{\gamma}$, while other centered on HeI, HeII, Si II, and Si III lines performed less satisfactorily, given the intrinsic weakness of these lines and the limited $\mathrm{S} / \mathrm{N}$ of several of our spectra.

The disentangled $\mathrm{H} \alpha$ profile for the tertiary component shown in Figs. 1 and 2 deserves some comments. The sharp absorptions caused by telluric $\mathrm{O}_{2}$ and $\mathrm{H}_{2} \mathrm{O}$ were intentionally not corrected for in the input spectra. The idea was to use them to test the performance of the SPD procedure. In fact, the wide orbit of the tertiary component around the eclipsing pair (for which we derive a period of $54.9 \pm 2.2 \mathrm{yr}$, an eccentricity of the wide orbit of $0.800 \pm 0.001$ and a time of periastron passage of $2455343 \pm 10$ day, all in good agreement with previous studies), is so slow that from the point of view of SPD the barycenter of the tertiary component is essentially at rest, when compared with the large orbital modulation of the component of the eclipsing pair. The total amplitude of the shift on the telluric lines imposed by the heliocentric correction is $46 \mathrm{~km} \mathrm{~s}^{-1}$, which is similar to the $50 \mathrm{~km} \mathrm{~s}^{-1}$ amplitude of the orbital motion of the tertiary star around its unseen companion (Lorenz et al. 1998). From the point of view of the SPD, they are out of phase with the 
E. Tamajo et al.: Asiago eclipsing binaries program. IV.

Table 4. Results of NLTE fitting, with the code GENFITT, of the Balmer lines on disentangled spectra of SZ Cam.

\begin{tabular}{|c|c|c|c|c|c|c|c|c|c|c|c|c|c|}
\hline Line & $\begin{array}{r}T_{\text {eff } 1} \\
(\mathrm{~K})\end{array}$ & $\begin{array}{r}\log g_{1} \\
{[\mathrm{cgs}]}\end{array}$ & $\begin{array}{r}v \sin i_{1} \\
\left(\mathrm{~km} \mathrm{~s}^{-1}\right)\end{array}$ & $\mathrm{ri}_{1}$ & $\begin{array}{r}T_{\text {eff } 2} \\
(\mathrm{~K})\end{array}$ & $\begin{array}{r}\log g_{2} \\
{[\operatorname{cgs}]}\end{array}$ & $\begin{array}{r}v \sin i_{2} \\
\left(\mathrm{~km} \mathrm{~s}^{-1}\right)\end{array}$ & $\mathrm{ri}_{2}$ & $\begin{array}{r}T_{\text {eff } 3} \\
(\mathrm{~K})\end{array}$ & $\begin{array}{r}\log g_{3} \\
{[\mathrm{cgs}]}\end{array}$ & $\begin{array}{r}v \sin i_{3} \\
\left(\mathrm{~km} \mathrm{~s}^{-1}\right)\end{array}$ & $\mathrm{ri}_{3}$ & $\chi_{v}^{2}$ \\
\hline \multicolumn{14}{|c|}{ NLTE not constrained by orbital solution } \\
\hline $\mathrm{H}_{\alpha}$ & $\begin{array}{r}30310 \\
\pm 190\end{array}$ & $\begin{array}{r}3.612 \\
\pm 0.013\end{array}$ & $\begin{array}{r}144.1 \\
2.8\end{array}$ & $\begin{array}{r}0.444 \\
\pm 0.012\end{array}$ & $\begin{array}{r}28260 \\
\pm 200\end{array}$ & $\begin{array}{r}3.867 \\
\pm 0.019\end{array}$ & $\begin{array}{r}117.9 \\
3.1\end{array}$ & $\begin{array}{r}0.301 \\
\pm 0.014\end{array}$ & $\begin{array}{r}26635 \\
\pm 216\end{array}$ & $\begin{array}{r}4.223 \\
\pm 0.031\end{array}$ & $\begin{array}{r}89.9 \\
3.7\end{array}$ & $\begin{array}{r}0.255 \\
\pm 0.014\end{array}$ & 0.745 \\
\hline $\mathrm{H}_{\beta}$ & $\begin{array}{r}30360 \\
\pm 190\end{array}$ & $\begin{array}{r}3.598 \\
\pm 0.016\end{array}$ & $\begin{array}{r}143.2 \\
3.6\end{array}$ & $\begin{array}{r}0.442 \\
\pm 0.017\end{array}$ & $\begin{array}{r}28420 \\
\pm 220\end{array}$ & $\begin{array}{r}3.793 \\
\pm 0.028\end{array}$ & $\begin{array}{r}117.1 \\
3.3\end{array}$ & $\begin{array}{r}0.304 \\
\pm 0.015\end{array}$ & $\begin{array}{r}26946 \\
\pm 229\end{array}$ & $\begin{array}{r}4.208 \\
\pm 0.036\end{array}$ & $\begin{array}{r}89.7 \\
4.5\end{array}$ & $\begin{array}{r}0.254 \\
\pm 0.017\end{array}$ & 0.868 \\
\hline $\mathrm{H}_{\gamma}$ & $\begin{array}{r}30400 \\
\pm 250\end{array}$ & $\begin{array}{r}3.586 \\
\pm 0.036\end{array}$ & $\begin{array}{r}142.9 \\
4.2\end{array}$ & $\begin{array}{r}0.396 \\
\pm 0.058\end{array}$ & $\begin{array}{r}28190 \\
\pm 300\end{array}$ & $\begin{array}{r}3.856 \\
\pm 0.032\end{array}$ & $\begin{array}{r}116.5 \\
4.2\end{array}$ & $\begin{array}{r}0.292 \\
\pm 0.053\end{array}$ & $\begin{array}{r}26854 \\
\pm 268\end{array}$ & $\begin{array}{r}4.212 \\
\pm 0.054\end{array}$ & $\begin{array}{r}88.7 \\
5.4\end{array}$ & $\begin{array}{r}0.312 \\
\pm 0.024\end{array}$ & 1.204 \\
\hline \multicolumn{14}{|c|}{ NLTE constrained with $\log g_{1}$ and $\log g_{2}$ from orbital solution } \\
\hline $\mathrm{H}_{\alpha}$ & $\begin{array}{r}30225 \\
\pm 205\end{array}$ & $\begin{array}{r}3.714 \\
\text { fixed }\end{array}$ & $\begin{array}{r}143.5 \\
\pm 2.9\end{array}$ & $\begin{array}{r}0.431 \\
\pm 0.015\end{array}$ & $\begin{array}{r}28295 \\
\pm 210\end{array}$ & $\begin{array}{r}3.820 \\
\text { fixed }\end{array}$ & $\begin{array}{r}117.6 \\
\pm 3.0\end{array}$ & $\begin{array}{r}0.308 \\
\pm 0.016\end{array}$ & $\begin{array}{r}26589 \\
\pm 220\end{array}$ & $\begin{array}{r}4.239 \\
\pm 0.035\end{array}$ & $\begin{array}{r}89.8 \\
\pm 3.8\end{array}$ & $\begin{array}{r}0.261 \\
\pm 0.015\end{array}$ & 0.814 \\
\hline $\mathrm{H}_{\beta}$ & $\begin{array}{r}30195 \\
\pm 220\end{array}$ & $\begin{array}{r}3.714 \\
\text { fixed }\end{array}$ & $\begin{array}{r}142.9 \\
\pm 3.4\end{array}$ & $\begin{array}{r}0.433 \\
\pm 0.018\end{array}$ & $\begin{array}{r}28355 \\
\pm 225\end{array}$ & $\begin{array}{l}3.820 \\
\text { fixed }\end{array}$ & $\begin{array}{r}116.9 \\
\pm 3.4\end{array}$ & $\begin{array}{r}0.309 \\
\pm 0.017\end{array}$ & $\begin{array}{r}26895 \\
\pm 235\end{array}$ & $\begin{array}{r}4.224 \\
\pm 0.041\end{array}$ & $\begin{array}{r}89.4 \\
\pm 4.6\end{array}$ & $\begin{array}{r}0.258 \\
\pm 0.019\end{array}$ & 0.984 \\
\hline $\mathrm{H}_{\gamma}$ & $\begin{array}{r}30209 \\
\pm 270\end{array}$ & $\begin{array}{r}3.714 \\
\text { fixed }\end{array}$ & $\begin{array}{r}142.1 \\
\pm 3.9\end{array}$ & $\begin{array}{r}0.422 \\
\pm 0.042\end{array}$ & $\begin{array}{r}28265 \\
\pm 315\end{array}$ & $\begin{array}{r}3.820 \\
\text { fixed }\end{array}$ & $\begin{array}{r}115.2 \\
\pm 4.1\end{array}$ & $\begin{array}{r}0.305 \\
\pm 0.047\end{array}$ & $\begin{array}{r}26930 \\
\pm 276\end{array}$ & $\begin{array}{r}4.194 \\
\pm 0.059\end{array}$ & $\begin{array}{r}88.6 \\
\pm 5.2\end{array}$ & $\begin{array}{r}0.273 \\
\pm 0.032\end{array}$ & 1.324 \\
\hline
\end{tabular}

Notes. The last column gives the reduced $\chi^{2} \cdot \mathrm{ri}_{1}, \mathrm{ri}_{2}$ and $\mathrm{ri}_{3}$ are the relative intensities of the three components to the given line $\left(\mathrm{ri}_{1}+\mathrm{ri}_{2}+\mathrm{ri}_{3}=1.0\right)$.

orbital motion of the main eclipsing pair and their radial velocity amplitudes are negligible in comparison. Thus the only effect impinged on the line profiles of the tertiary star in Fig. 2 is a moderate broadening of the stellar and telluric lines (the rotational velocity below derived for the tertiary star is corrected for this smearing). It is reassuring that the SPD procedure attributed the telluric absorptions to the tertiary component, cleaning them completely from the disentangled spectra of the primary and secondary components (cf. Hadrava 2006). Particularly interesting in this respect is the presence, on the $\mathrm{H} \alpha$ profile of the tertiary component in Figs. 1 and 2, of the sharp telluric $\mathrm{H}_{2} \mathrm{O}$ line at exactly the expected rest wavelength of $6564.21 \AA$, even if this line is well within the $\mathrm{H} \alpha$ profile and just on the red of its photocenter.

It is worth noticing that the overall disentangled $\mathrm{H} \alpha$ profile for the tertiary star in Fig. 1 differs significantly from the shape of the same line for the two other stars. For sake of discussion, we could describe it as the apparent superposition of a broader and a narrower components. Could it be so because our SPD procedure has also effectively deconvolved the two components making up the third star binary? We plan to investigate this possibility elsewhere by, in a tailored analysis combining other spectroscopic and photometric data we are planning to obtain for this specific purpose.

\section{Orbital solution}

A photometric solution for SZCam was obtained with the WD code (Wilson \& Devinney 1971; Wilson 1998) in its WD98K93d version as developed by Milone et al. (1992), by adopting the MODE-2 option, appropriate for detached binary stars, which implements Roche geometry and a detailed treatment of reflection and other physical phenomena. It adopted the mass ratio as provided by the spectral disentangling. We used the version of the standard differential corrections procedure (WDDC) and iterated until all parameter corrections were less than their formal errors. The orbital solution was then refined by incorporating the information on the radial velocity semi-amplitudes derived by the spectral disentangling, so that

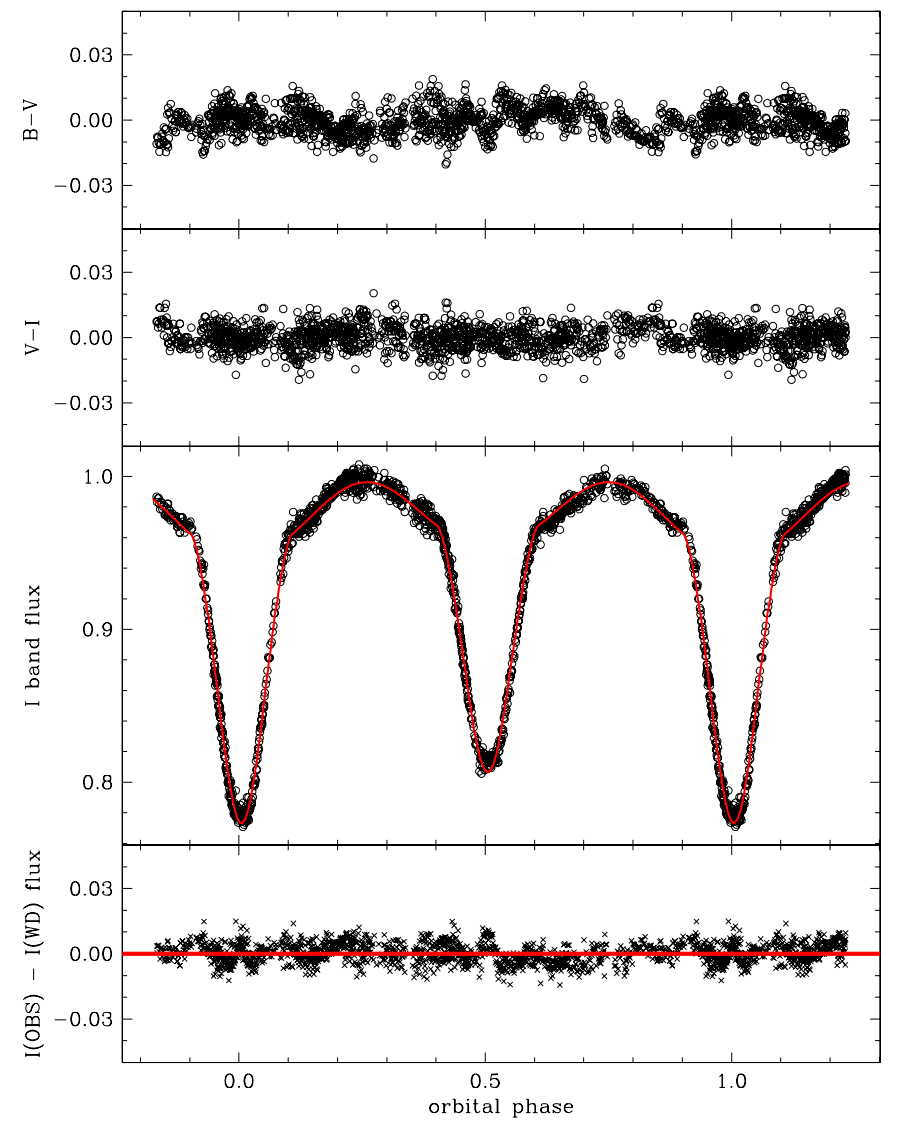

Fig. 3. The orbital solution as given in Table 5 is overplotted on our photometric data from Table 3. The middle panel shows the $I_{\mathrm{C}}$ band light curve and the other three panels presents the residuals in $(B-V)$, $\left(V-I_{\mathrm{C}}\right)$ and $I_{\mathrm{C}}$ (top to bottom, respectively).

the radial velocity curve generated by the WD code matched that obtained from the spectral disentangling.

The full set of fixed and controlled orbital parameters are given in Table 5, where the given errors are those formal to the WD solution (the true errors are probably larger, in particular 


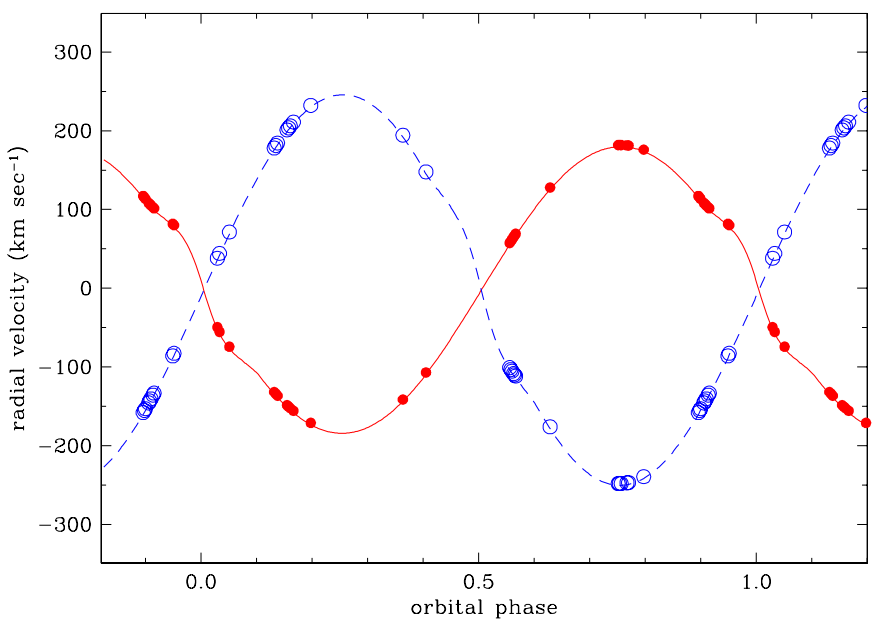

Fig. 4. The position in phase of the spectroscopic data used in the disentangling is overplotted on the radial velocity curves from the orbital solution of Table 5. Filled red circles mark the hotter and more massive (primary) star, and the open blue circles the cooler and less massive (secondary) star.

Table 5. Light-curve solution, where the given errors are "formal errors" due to the WD solution.

\begin{tabular}{|c|c|c|c|}
\hline Parameter & Value & & Error \\
\hline period (days) & 2.6983845 & \pm & 0.0000011 \\
\hline$t_{\circ}($ primary ecl. HJD $)$ & 2455462.5310 & \pm & 0.00023 \\
\hline$a\left(R_{\odot}\right)$ & 23.97 & \pm & 0.15 \\
\hline$i\left(^{\circ}\right)$ & 75.16 & \pm & 0.39 \\
\hline$e$ & 0.00 & & \\
\hline$T_{1}(\mathrm{~K})$ & 30360 & \pm & (fixed) \\
\hline$T_{1}-T_{2}(\mathrm{~K})$ & 3116 & \pm & 255 \\
\hline$\Omega_{1}$ & 3.909 & \pm & 0.026 \\
\hline$\Omega_{2}$ & 3.592 & \pm & 0.025 \\
\hline$R_{1}\left(R_{\odot}\right)$ & 8.91 & \pm & 0.05 \\
\hline$R_{2}\left(R_{\odot}\right)$ & 6.70 & \pm & 0.12 \\
\hline$M_{1}\left(M_{\odot}\right)$ & 14.31 & \pm & 0.54 \\
\hline$M_{2}\left(M_{\odot}\right)$ & 10.69 & \pm & 0.38 \\
\hline$M_{\mathrm{bol}, 1}$ & -6.93 & \pm & 0.04 \\
\hline$M_{\mathrm{bol}, 2}$ & -6.28 & \pm & 0.06 \\
\hline $\log g_{1}(\mathrm{cgs})$ & 3.70 & \pm & 0.01 \\
\hline $\log g_{2}(\mathrm{cgs})$ & 3.82 & \pm & 0.02 \\
\hline$L_{1}(\text { Ic band })^{a}$ & 0.444 & \pm & 0.004 \\
\hline$L_{2}(\text { Ic band })^{a}$ & 0.301 & \pm & 0.005 \\
\hline$l_{3}(\mathrm{Ic} \text { band })^{b}$ & 0.255 & \pm & 0.010 \\
\hline$R_{1, \text { pole }}{ }^{c}$ & 0.341 & \pm & 0.004 \\
\hline$R_{1 \text { point }}{ }^{c}$ & 0.379 & \pm & 0.006 \\
\hline$R_{1, \text { side }}^{c}$ & 0.360 & \pm & 0.006 \\
\hline$R_{1 \text { back }}{ }^{c}$ & 0.371 & \pm & 0.005 \\
\hline$R_{2, \text { pole }^{c}}{ }^{2}$ & 0.273 & \pm & 0.005 \\
\hline$R_{2, \text { point }}{ }^{c}$ & 0.300 & \pm & 0.008 \\
\hline$R_{2, \text { side }}{ }^{c}$ & 0.276 & \pm & 0.008 \\
\hline$R_{2, \text { back }}{ }^{c}$ & 0.274 & \pm & 0.008 \\
\hline
\end{tabular}

Notes. ${ }^{(a)}$ Relative light contributions $\left(L_{1}+L_{2}+l_{3}=1\right)$; ${ }^{(b)}$ fractional contribution of the third light at maximum; ${ }^{(c)}$ fractional Roche radii in units of separation of mass centers.

considering that the eclipses are only partial and not total). We caution that the formal errors can be optimistic when there are strong correlations between parameters, so should be considered with care (e.g. Southworth et al. 2004; Southworth et al. 2007).

The orbital solution is overplotted to the observed light curve in Fig. 3. For our modeling, we adopted temperature of the primary star as derived by the NLTE analysis, and the bolometric

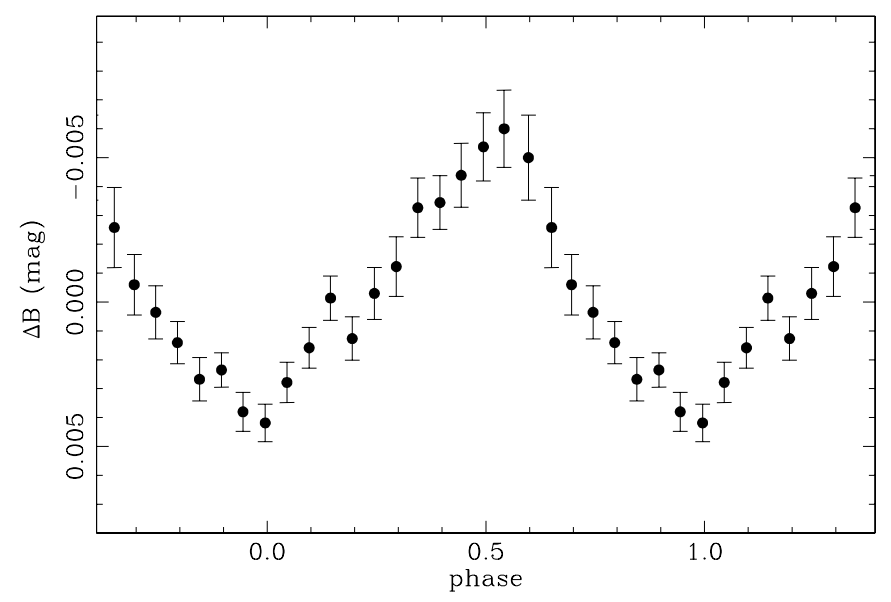

Fig. 5. The light curve of the $\beta$ Cephei star in SZCam, with a period of 0.33265 days and a total amplitude of $0.0105 \mathrm{mag}$.

albedos and gravity brightening exponents appropriate for radiative atmospheres (Claret 2001, 1998), and limb darkening coefficients from Van Hamme (1993). The final solution shows a negligible dependence on the way limb darkening is accounted for, but it is very sensitive to the strength of the third light.

The contribution of third light implied by the orbital solution is $25.5 \%$, and $26.0 \%$ from NLTE modeling of line-profiles (both methods to the same wavelength region, the $V$ band, whose effective wavelength is matched by the weighted average of the NLTE results based on $\mathrm{H}_{\gamma}, \mathrm{H}_{\beta}$ and $\mathrm{H}_{\alpha}$ lines). The two methods therefor converge on the same contribution of third light within $0.5 \%$. To test the effect of uncertainty on the amount of third light, we reran the WD solution by first increasing the third light for $+0.5 \%$ and then decreasing it by the same amount (i.e. imposing $l_{3}$ first to $25.0 \%$ and then to $26.0 \%$ ). The effect of such a $+0.5 \%$ change in the contribution of the third light reflects in a change of $T_{\text {eff }}$ in the secondary component by $1.1 \%$, and a change of $1.2 \%$ in the radii.

Thus, the order of magnitude of the difference in $T_{\text {eff }}$ between spectroscopy (NLTE modeling) and photometry (WD calculation) are a few hundred kelvins. A discussion of the temperature scale of hot stars is given in Sect. 5 .

Our final solution adopted the linear limb darkening law and converged on the following limb-darkening parameters: $\mathrm{x} 1($ bolo $)=0.500, \mathrm{x} 2($ bolo $)=0.500$, and the monochromatic ones as $\mathrm{x} 1(\mathrm{mono})=0.301, \mathrm{x} 2(\mathrm{mono})=0.207$ for the primary and secondary components, respectively.

\subsection{Reddening and distance}

The mean $E_{B-V}$ color-excess of NGC 1502 amounts to about 0.75 mag according to Reimann \& Pfau (1987) and $E_{B-V}=0.70$ following Dias et al. (2002) with a possible variation of $\Delta E_{B-V}=0.2$ over the area of the cluster (Janes \& Adler 1982; Yadav \& Sagar 2001). The reddening law along the line of sight to NGC 1502 deviates from the standard $A_{V}=3.1$, because it is shifted toward $A_{V}=2.6$ (Tapia et al. 1991; Pandey et al. 2003; Weitenbeck et al. 2008). Averaging member stars listed by the WEBDA database from the sources of $B, V$ photometry for NGC 1502 we built the color-magnitude diagram of Fig. 6, where the fit is carried out for a Padova isochrone of solar metallicity and $10 \mathrm{Myr}$, scaled in distance and extinction. The position of member stars in the diagram is so scattered that the fitting with an isochrone is not well constrained. The scatter is intrinsic to the 
E. Tamajo et al.: Asiago eclipsing binaries program. IV.

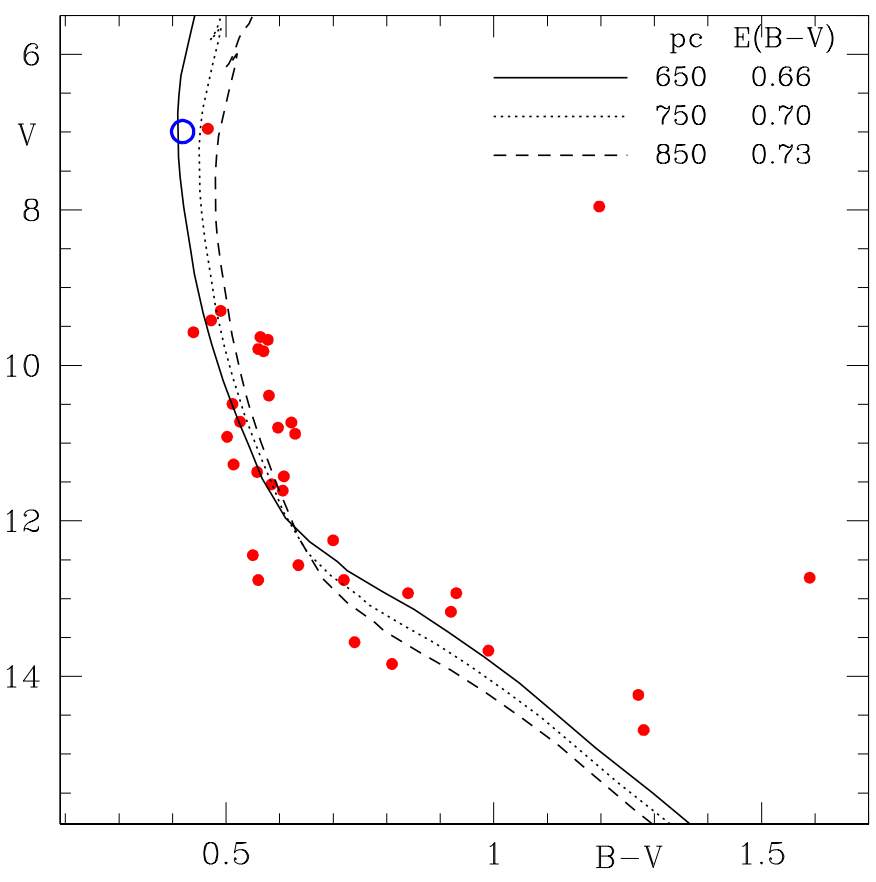

Fig. 6. Color magnitude diagram of the open cluster NGC 1502 built from photometric data retrieved from WEBDA database (http : / www . univie.ac.at/webda/). The blue open circle marks SZCam. The lines are a Padova solar composition isochrone for an age of $10 \mathrm{Myr}$, shifted according to indicated reddening and distances.

members of the cluster, as illustrated by the similarly dispersed color-magnitude diagram published by Michalska et al. (2009), who tentatively find $E_{B-V}=0.70$ and $1 \mathrm{kpc}$ distance from their own photometric data for NGC 1502.

The equivalent width of interstellar lines correlates with reddening very well. To check on the reddening to be assumed for SZ Cam in computing its distance, we measured the interstellar lines appearing on our REOSC Échelle spectra. The NaI D1,2 lines are clearly saturated, indicating a reddening $E_{B-V}>0.55$, while KI $7699 \AA$ has an equivalent width of $0.19 \AA$ that corresponds to $E_{B-V}=0.75$ following the calibrations of Munari $\&$ Zwitter (1997). In the following, we assume the mean value $E_{B-V}=0.73$ as the reddening affecting SZ Cam.

To compute a distance to SZ Cam from the orbital solution, we adopted a bolometric correction $\mathrm{BC}=-3.16$ from Bessell et al. (1998) for all three components, and for the Sun $L_{\odot}=$ $3.826 \times 10^{26} \mathrm{~W}$ and $M_{\mathrm{bol}, \odot}=4.74$. The classical method of determining the distance to an eclipsing binary is to calculate the luminosity of each component star from its radius and effective temperature. The resulting values of absolute bolometric magnitude, $M_{\mathrm{bol}}$, are then converted to absolute visual magnitudes, $M_{\mathrm{V}}$, using bolometric corrections (BCs). The combined $M_{\mathrm{V}}$ of the components is then compared to the apparent visual magnitude $m_{\mathrm{V}}$ to find the distance modulus (e.g. Harmanec \& Prša 2011; Southworth et al. 2005).

We obtained a distance of $870 \pm 30 \mathrm{pc}$ for SZCam. This lies comfortably close to the rather uncertain value for the distance of the parent NGC 1502 cluster. Unfortunately, this determination cannot be checked against the Hipparcos parallax for SZ Cam, which is useless since its error is six times more than the value (Van Leeuwen 2007).

\section{2. $\beta$ Cep pulsation}

The high accuracy of our photometry allows for searching and characterizing the low-amplitude intrinsic variability of the individual stars making up SZ Cam. The presence of a stable and periodic variability with a total amplitude of around $0.01 \mathrm{mag}$ and a period of about eight hours is already evident in our data by looking at the individual long photometric observing runs we collected on SZ Cam, which lasted for many consecutive hours. This microvariability takes the form of a sinusoidal-like disturbance around the light curve modulated by eclipses.

To isolate this variability, we subtracted the flux corresponding to the orbital light curve at that phase from each observation, thus working on the residuals. The residuals were then searched for periodicity using the Deeming-Fourier algorithm for unequally spaced data (Deeming 1975), which led again to a very strong peak at $0.33265 \pm 0.00005$ days $\left(7^{\mathrm{h}} 59^{\mathrm{m}}\right)$. The corresponding light curve is presented in Fig. 5. To plot the latter, we took the residuals from the orbital light curve for all 1517 observations, divided the 0.33265 day period into 20 bins, and plotted the mean magnitude and its sigma for each bin. In this way the light curve is much cleaner than when plotting all 1517 individual points. The observed total amplitude is $\Delta B=0.0105$ $( \pm 0.0005) \mathrm{mag}$, and the corresponding ephemeris is

$\min (B)=2455311.140( \pm 0.002)+0.33265( \pm 0.00005) \times E$.

Such a low-amplitude variability, its sinusoidal light curve, and the length of the period are typical of $\beta$ Cep stars. According to the catalog of $\beta$ Cep stars of Stankov \& Handler (2005, hereafter ST05), their spectral type range from B0 to B3 and the luminosity class from I to V, with two thirds of the total being composed by B1 and B2 stars of luminosity classes III, IV, and V. Their variability is due to pulsations driven by the $\kappa^{-}$ mechanism (Maeder 2009; Dziembowski \& Pamyatnykh 1993; Sterken \& Jerzykiewicz 1993; Moskalik \& Dziembowski 1992), and the general aspect of their light curves is similar to a sinusoid (Hoffmeister 1985; Sterken \& Jaschek 1996). They are rare objects, since the ST05 catalog listing only 93 validated $\beta$ Cep stars. The median value of their pulsation amplitude is $0.02 \mathrm{mag}$, and the frequency distribution of the pulsation periods is a broad Gaussian, centered at 0.171 days, with the shortest cataloged period 0.0667 days (V945 Sco) and the longest 0.319 days (V595 Per). According to the ST05 catalog, only four $\beta$ Cep stars are eclipsing binaries ( $\eta$ Ori, HD 92024, HIP 84655 , and EN Lac), and only one of them is also a triple system ( $\eta$ Ori).

In this context, SZ Cam stands out as a very interesting member of the $\beta$ Cep group of stars, because it has the longest known pulsation period, it is eclipsing, it is a quadruple system, and it is also member of a young open cluster. The bottom panel of Fig. 3 suggests that neither at primary nor at secondary eclipse is the "noise" in the light curve due to the $\beta$ Cep pulsation reduced. This seems to indicate that the $\beta$ Cep variable is the primary component of the non-eclipsing pair in the quadruple system SZCam. The spectral type of this star (inferred from the other observable properties) should be B1V, making it a perfect match to the average spectral type of known $\beta$ Cep variables.

\section{Atmospheric analysis and comparison with stellar models}

We performed NLTE analysis of the Balmer lines on the disentangled spectra of the individual components of SZ Cam. These are the strongest lines in their spectra, and we refrained from 
using others lines with noisier profiles because little gain would have been achieved. In OB stars, numerous oxygen, nitrogen, and silicon lines, are superimposed on the Balmer-lines are superimposed, so we carefully selected parts of their profiles that are free of such blendings as suitable for matching to theoretical line profiles. The code GENFITT has been developed to fit theoretical line profile to hydrogen lines by $\chi^{2}$ minimization in $T_{\mathrm{eff}}$ (Tamajo et al. 2011).

We used a grid of theoretical NLTE spectra that were computed for O-stars over $T_{\text {eff }}=27500-55000 \mathrm{~K}$ in steps of $2500 \mathrm{~K}$ and $\log g=3.00-4.75 \mathrm{dex}$ in steps of $0.25 \mathrm{dex}$, and for B-stars over $T_{\text {eff }}=15000-30000 \mathrm{~K}$ in steps of $1000 \mathrm{~K}$ and $\log g=1.75-4.75$ dex also in steps of 0.25 dex. The results we obtained are summarized in the upper half of Table 4 ("NLTE not constrained by orbital-solution") and the theoretical line profile is overplotted to the observed one in Fig. 2. A warning is necessary here about the reported results on the third component. The results in Table 4 come from treating the disentangled line profiles of the third star as if it were a single star. However, in discussing Fig. 1 in Sect. 3.2, we consider that our disentangling could have come close to resolve the tertiary component into the two stars that compose the SB1 non-eclipsing pair in SZCam. Therefore, the line we modeled as coming from a single star could actually be the blend of the lines of the two components of the SB1 non-eclipsing pair.

The risk of degeneracy between the atmospheric parameters is always present when analyzing stellar spectra. To reduce it, we reran the NLTE analysis by fixing $\log g$ for the primary and secondary star to the value provided by the orbital solution in Table 5. The results are summarized in the lower half of Table 4. The differences between the two sets of NLTE results are minimal, which reinforces confidence in them. The parameter changing the most is the surface gravity of the primary star, showing a difference of mere $\Delta \log g=0.1$ dex.

Regarding the effective temperature $\left(T_{\text {eff }}\right)$, its determination in OB stars is a complex task (e.g. Böhm-Vitense 1981; Crowther 1998). Given the need for a detailed treatment of non-LTE effects and the presence of stellar winds (Kudritzki \& Hummer 1990), satisfactory modeling of such atmospheres that includes the effects of numerous metal-lines remains difficult to achieve (cf. Schaerer \& Schmutz 1994; Hillier \& Miller 1998; Pauldrach et al. 2001). Most of published spectral analysis have so far been based on simple non-LTE models. For example, the calibration of stellar parameters of $\mathrm{O}$ and early B type stars of Vacca et al. (1996) is based only on results from static, plane parallel, pure hydrogen, and helium $(\mathrm{H}-\mathrm{He})$ non-LTE models. Their derived temperature scale for $\mathrm{O}$ stars is found to be significantly hotter than most earlier calibrations. Such simplifications non-negligibly affect the estimated fundamental parameters of $\mathrm{O}$ stars (e.g. luminosities, Lyman continuum fluxes, etc.). Accurate calibrations of hot stars are crucial for various astrophysical topics, such as comparisons with stellar evolution models (and the effects of mass loss and rotation), determinations of the initial mass function and ages of clusters, ionization balance of HII regions, and supernova progenitors.

For a given position of $\mathrm{OB}$ stars in the HR diagram, the difference in mass between theoretical models and orbital solutions can be fairly large, for example on the order of $2 M_{\odot}$ for the binary systems V478 Cyg (Popper \& Hill 1991) and EM Car (Andersen \& Clausen 1989) even more than $10 M_{\odot}$ for the system HD 152248 (Penny et al. 1999). Because this involves the transformation from the theoretical HR diagram ( $L_{\text {bol }}$ vs. $\left.T_{\text {eff }}\right)$ to the observed color-magnitude one (particularly in the $u v b y \beta$ system), some of the discrepancy can be ascribed to uncertainties in involved distance, reddening, observed photometry, its transformation to $T_{\text {eff }}$ (leading up to $\pm 1000-2000 \mathrm{~K}$ uncertainties), and the bolometric correction.

The bolometric correction (BCs) is a very steep function of the assumed effective temperature $\left(T_{\text {eff }}\right)$. For O-stars, a $10 \%$ error in $T_{\text {eff }}$ results in an error of $30 \%$ in the derived bolometric luminosity of a star (see Massey 1998), severely affecting the attempt to use stellar evolutionary tracks to determine distances, initial mass functions, and ages of clusters (see, Massey 1998, 2005; Slesnick et al. 2002). In addition, a $10 \%$ uncertainty in $T_{\text {eff }}$ results in a factor of 2 or more uncertainty in the Lyman continuum flux, affecting our understanding of the ionization balance of HII regions and the porosity of the interstellar medium in general (see Oey \& Kennicutt 1997; Oey 2005).

The rotational velocities derived in Table 4 from NLTE analysis of Balmer lines confirmed the profiles of the SiIII lines at 4553, 4568, and $4575 \AA$, from which we derived $145 \mathrm{~km} \mathrm{~s}^{-1}$, $119 \mathrm{~km} \mathrm{~s}^{-1}$, and $90 \mathrm{~km} \mathrm{~s}^{-1}$, for the primary, secondary, and tertiary components, respectively. These values are just $1.3 \%$ higher than those derived from Balmer lines. In view of the relative intensity of SiIII and Balmer lines, in the rest of this paper we adopt the rotational velocities from Balmer lines. From the orbital solution in Table 5, the expected corotation velocities would be $167 \mathrm{~km} \mathrm{~s}^{-1}$ for the primary and $126 \mathrm{~km} \mathrm{~s}^{-1}$ for the secondary. The secondary essentially match within errors the condition of corotation, while the primary one, which is already evolved away from the main sequence, is rotating slower than corotation.

For the third component, assuming it is corotating with the 2.798 day orbital period of the SB1 non-eclipsing pair, the $89 \mathrm{~km} \mathrm{~s}^{-1}$ projected rotational velocity (cf. Table 4) would correspond to a radius of $R \sim 5 \sin i R_{\odot}$. This is close to the value of $\sim 5.5 R_{\odot}$ expected for a star of the same temperature and luminosity of the tertiary in SZ Cam. This seems to indicate that the orbital inclination for the SB1 non-eclipsing pair is relatively large, too.

Figures 7 and 8 compare the position of the three components of SZCam on a temperature-gravity plane with Geneva theoretical isochrones from Meynet et al. (1993) and stellar tracks from Ekström et al. (2008), which include mass loss and effect of rotation. These tracks and isochrones, which are computed for single stars, do not match the properties of the components of SZ Cam as accurately constrained by the analysis presented in this paper. In Fig. 7 a single isochrone cannot pass through all three stars at the same time, and the masses inferred by the stellar tracks in Fig. 8 are much higher than those provided by the orbital solution. Similar mismatches are common for eclipsing binaries containing young, massive stars, and are discussed at length in Hilditch (2004).

The evident mismatch probably arises from a combination of stellar models that would benefit from improvement, and from the effect of SZCam components as members of close pairs. Evolution of fast-rotating and heavily mass- losing stars, trapped in close binary systems, is arguably different from that of isolated, single counterparts.

The temperatures and gravities of primary and secondary stars are obtained from a weighted average of values from the orbital solution and the NLTE analysis. The radius and bolometric magnitude for the third star are estimated under the assumption that it is corotating in the SB1 non-eclipsing binary (see Sect. 5). However, see Sect. 5 for a discussion of the difference between method precision and true uncertainties for hot stars. 
E. Tamajo et al.: Asiago eclipsing binaries program. IV.

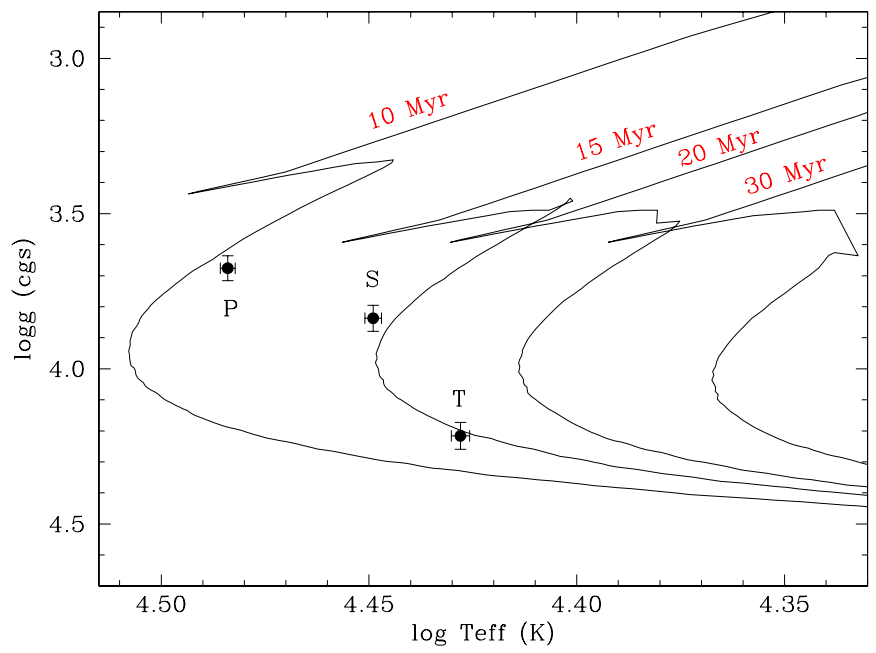

Fig. 7. Comparison of the $\log T_{\text {eff }}-\log g$ plane between the observed values for the components of SZ Cam and the theoretical isochrones of the Geneva group (Meynet et al. 1993), which include the effect of stellar rotation and mass loss. Labels $\mathrm{P}, \mathrm{S}$, and T mark the primary, secondary and tertiary components respectively. The size of the error bars corresponds to the formal errors of the orbital solution in Table 5 .

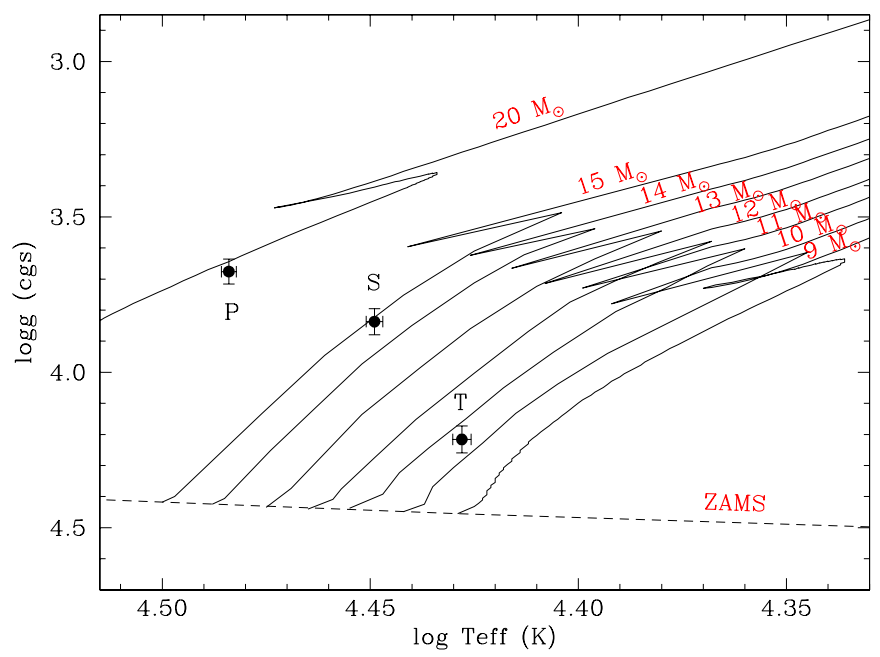

Fig. 8. Comparison of the $\log T_{\text {eff }}-\log g$ plane and the observed values for the components of SZ Cam and the theoretical stellar tracks of the Geneva group (Ekström et al. 2008), which include the effect of stellar rotation and mass loss. Labels P, S, and T mark the primary, secondary, and tertiary components respectively. The size of the error bars corresponds to the formal errors of the orbital solution in Table 5.

\section{Summary}

The input data for the analysis presented in this paper were a set of $1517 B V I_{\mathrm{C}}$ observing sequences obtained in 43 different observing nights, and 40 high-resolution REOSC Échelle spectra, well distributed in orbital phase and varied in S/N.

In addition to accurately mapping the eclipse light curve, the extreme accuracy of the photometric data, has for the first time revealed the presence of a $\beta$ Cep variable in the SZCam hierarchical system, probably located within the non-eclipsing SB1 pair. The pulsation period is 0.33265 days and the observed total amplitude in the $B$ band is $0.0105 \mathrm{mag}$. The composited REOSC Échelle spectra were subjected to spectral disentangling that returned the individual spectra of the two components of the eclipsing pair and of the primary of the non-eclipsing SB1 pair.
Table 6. Summary of the main properties of SZCam.

\begin{tabular}{|c|c|c|c|c|}
\hline Parameter & System & Primary & Secondary & Tertiary \\
\hline$E(B-V)$ & $0.73 \pm 0.05$ & & & \\
\hline dist. (pc) & $870 \pm 30$ & & & \\
\hline$a \mathrm{SB} 2\left(R_{\odot}\right)$ & $23.97 \pm 0.15$ & & & \\
\hline $\operatorname{mass}\left(M_{\odot}\right)$ & & $14.31 \pm 0.54$ & $10.69 \pm 0.38$ & \\
\hline radiu & & & 6.7 & (5.0) \\
\hline$T_{\text {eff }}($ & & 3032 & 280 & 26760 \\
\hline $\log g$ & & $3.70 \pm 0.01$ & $3.82 \pm$ & $4.22 \pm 0.03$ \\
\hline$v \sin i$ & & $145 \pm 2.3$ & $119 \pm 2.8$ & \\
\hline$M_{\mathrm{bol}}(\mathrm{mag})$ & & $-6.93 \pm 0.04$ & $-6.28 \pm 0.06$ & $(-5.44)$ \\
\hline
\end{tabular}

The disentangled spectra provided the radial velocity amplitudes of the relative orbital motions, and were also NLTE-modeled to derive the temperature, surface gravity, and projected rotational velocities of all components.

This set of data was modeled with the Wilson-Devinney code, and the orbit of the eclipsing pair was derived. Our orbital solution, while confirming previous results, significantly improves on the associated formal errors, and for the parameters in common it is accurately confirmed by the results of the NLTE spectral analysis. For the eclipsing pair, the radius of the primary is $37 \%$ of the orbital separation, $28 \%$ for the secondary. Noteworthy is that the relative contribution of the tertiary star to the whole system brightness turned out to be the same.

The comparison with theoretical isochrones that incorporates mass-loss and rotation but which are computed for single, isolated stars, do not match the properties of the components closely of the SZCam hierarchical system, which are evolving in a close binary configuration. This comparison would nevertheless suggest that SZ Cam is younger than $\sim 20 \mathrm{Myr}$, with the primary and secondary stars well away from the ZAMS, and the tertiary closer to it.

We hope in the near future to obtain higher resolution and $\mathrm{S} / \mathrm{N}$ spectra of this interesting object, with the aim of further constraining the nature and orbit of the non-eclipsing SB1 pair performing a detailed NLTE chemical analysis of all its components. The chemical adundances should convincingly constrain the age and mass loss of the components.

\section{References}

Andersen, J. 1991, A\&ARv, 3, 91

Andersen, J., \& Clausen, J. V. 1989, A\&A, 213, 183

Balega, I. I., Balega, Yu. Yu, \& Maximov, A. F. 2007, Astrophys. Bull., 62, 339 Bessell, M., Castelli, F., \& Plez, B. 1998, A\&A, 333, 231

Böhm-Vitense, E. 1981, ARA\&A, 19, 295

Budding, E. 1975, Ap\&SS, 36, 329

Chochol, D. 1980, Bulletin of the Astronomical Institutes of Czechoslovakia, 31, 321

Claret, A. 1998, A\&AS, 131, 395

Claret, A. 2001, MNRAS, 327, 989

Crowther, P. A. 1998, IAU Symp., 189, 137

Deeming, T. J. 1975, Ap\&SS, 36, 137

Dias, W. S., Alessi, B. S., Moitinho, A., \& Lépine, J. R. D. 2001, A\&A, 389, 871 Dziembowski, W., \& Pamyatnykh, A. 1993, MNRAS, 262, 204

Ekström, S., Meynet, G., Maeder, A., \& Barblan, F. 2008, A\&A, 478, 467

Gorda, S. Y. 2000, Information Bulletin on Variable Stars, 4839, 1

Gorda, S. Y. 2008, Astron. Lett., 34, 316

Gorda, S. Y., \& Polushina, T. S. 1987, Astronomical-Geodetical Investigations, Statistical Methods, 96

Gorda, S. Yu., Balega, Yu. Yu., Pluzhnik, E. A., et al. 2007, Astrophys. Bull., 62, 352

Guthnick, P., \& Prager, R. 1930, Astron. Nachr., 239, 14

Hadrava, P. 1995, A\&AS, 114, 393

Hadrava, P. 2006, A\&A, 448, 1149

Harmanec, P., \& Prša, A. 2011, PASP, 123, 976 
Harries, T. J., Hilditch, R. W., \& Hill, G. 1998, MNRAS, 295, 386

Henden, A., \& Munari, U. 2006, A\&A, 458, 339

Hensberge, H., Pavlovski, K., \& Verschueren, W. 2000, A\&A, 358, 553

Hensberge, H., Ilijić, S., \& Torres, K. B. V. 2008, A\&A, 482, 1031

Hensberge, H., \& Pavlovski, K. 2007, in Binary stars as Critical Tools and Tests in Contemporary Astrophysics, ed. W. I. Hartkopf, E. F. Guinan, \& P. Harmanec (Cambridge: Cambridge Univ. Press), IAU Symp., 240, 136

Hilditch, R.W. 2004, in Spectroscopically and Spatially Resolving the Components of the Close Binary Stars, ed. R. W. Hilditch, H. Hensberge, K. Pavlovski (San Francisco: ASP), ASP Conf. Ser., 318, 111

Hillier, D. J., \& Miller, D. L. 1998, ApJ, 497, 407

Hoag, A. A. 1961, Publications of the US Naval Observatory, 2d ser., v. 17, pt. 7 (Washington: US Govt. Print. Off.), 344

Hoffmeister, C., Richter, G., \& Wenzel, W. 1985, Variable Stars (SpirngerVerlag)

Ilijić, S., Hensberge, H., Pavlovski, K., \& Freyhammer, L. M. 2004, in Spectroscopically and Spatially Resolving the Components of the Close Binary Stars, ed. R. W. Hilditch, H. Hensberge, \& K. Pavlovski, ASP Conf. Ser., 318, 111

Janes, K., \& Adler, D. 1982, ApJS, 49, 425

Kitamura, M., \& Yamasaki, A. 1971, Information Bulletin on Variable Stars, 582,1

Kudritzki, R. P., \& Hummer, D. G. 1990, ARA\&A, 28, 303

Lorenz, R., Mayer, P., \& Drechsel, H. 1998, A\&A, 332, 909

Maeder, A. 2009, Physics, Formation and Evolution of Rotating Stars (Springer)

Martins, F., Schaerer, D., \& Hillier, D. J. 2002, A\&A, 382, 999

Mason, B. D., Gies, D. R., Hartkopf, W. I., et al. 1998, AJ, 115, 821

Massey, P. 1998, in The Stellar Initial Mass Function, 38th Herstmonceux Conference, ed. G. Gilmore, \& D. Howell (San Francisco: ASP), ASP Conf. Ser., 142,17

Massey, P., Puls, J., \& Pauldrach, A. W. A. 2005, ApJ, 627, 477

Mayer, P., Lorenz, R., Chochol, D., et al. 1994, A\&A, 288, 13

Mayer, P., Drechsel, H., Kubát, J., \& Šlechta, M. 2010, A\&A, 524, A1

Meinel, A. B., Aveni, A. F., \& Stockton, M. W. 1968, Tucson, Optical Sciences Center and Steward Observatory, University of Arizona

Meynet, G., Mermilliod, J.-C., \& Maeder, A. 1993, A\&AS, 98, 477

Michalska, G., Kubát, J., Korčáková, D., et al. 2007, IAU Symp., 240, 555

Milone, E. F., Stagg, C. R., \& Kurucz, R. L. 1992, ApJS, 79, 123

Morgan, W. W., Code, A. D., \& Whitford, A. E. 1955, ApJS, 2, 41

Moskalik, P., \& Dziembowski, W. A. 1992, A\&A, 256, L5

Munari, U., \& Lattanzi, M. G. 1992, PASP, 104, 121

Munari, U., \& Zwitter, T. 1997, A\&A, 318, 269

Oey, M. S. 2005, in The Local Group as an Astrophysical Laboratory, ed. M. Livio (Cambridge: Cambrdige Univ. Press)

Oey, M. S., \& Kennicutt, R. C., Jr. 1997, MNRAS, 291, 827

Olsen, E. H. 1971, Information Bulletin on Variable Stars, 533, 1
Osterbrock, D. E., \& Martel, A. 1992, PASP, 104, 76

Osterbrock, D. E., Fulbright, J. P., Martel, A. R., et al. 1996, PASP, 108, 277 Pandey, A. K., Upadhyay, K., Nakada, Y., \& Ogura, K. 2003, A\&A, 397, 191 Pauldrach, A. W. A., Hoffmann, T. L., \& Lennon, M. 2001, A\&A, 375, 161

Pavlovski, K. 2004, in Spectroscopically and Spatially Resolving the Components of the Close Binary Stars (San Francisco: ASP), ed. R. W. Hilditch, H. Hensberge, \& K. Pavlovski, ASP Conf. Ser., 318, 206

Pavlovski, K., \& Hensberge, H. 2005, A\&A, 439, 309

Pavlovski, K., \& Hensberge, H. 2010, ASP Conf., 435, 207

Pavlovski, K., \& Southworth, J. 2009, MNRAS, 394, 1519

Pavlovski, K., Tamajo, E., Koubský, P., et al. 2009, MNRAS, 400, 791

Penny, L. R., Gies, D. R., \& Bagnuolo, W. G. 1999, ApJ, 518, 450

Plaskett, J. S. 1924, Publications of the Dominion Astrophysical Observatory Victoria, 2, 287

Polushina, T. S. 1977, Peremennye Zvezdy, 20, 473

Popper, D. M., \& Hill, G. 1991, AJ, 101, 600

Reimann, H.-G., \& Pfau, W. 1987, Astron. Nachr., 308, 111

Schaerer, D., \& Schmutz, W. 1994, A\&A, 288, 231

Simon, K. P., \& Sturm, E. 1994, A\&A, 281, 286

Simon, K. P., Sturm, E., \& Fiedler, A. 1994, A\&A, 292, 507

Siviero, A., Munari, U., Sordo, R., et al. 2004, A\&A, 417, 1083 (Paper I)

Slesnick, C. L., Hillenbrand, L. A., \& Massey, P. 2002, ApJ, 576, 880

Southworth, J., \& Clausen, J. V. 2007, A\&A, 461, 1077

Southworth, J., Maxted, P. F. L., \& Smalley, B. 2004, MNRAS, 351, 1277

Southworth, J., Maxted, P. F. L., \& Smalley, B. 2005, A\&A, 429, 645

Southworth, J., Bruntt, H., \& Buzasi, D. L. 2007, A\&A, 467, 1215

Stankov, A., \& Handler, G. 2005, ApJS, 158, 193

Sterken, C., \& Jaschek 1996, Lightcurves of Variable Stars (Cambridge Univ. Press)

Sterken, C., \& Jerzykiewicz, M. 1993, Space Sci. Rev., 62, 95

Tamajo, E. 2009, Ph.D. Thesis, Dept. of Physics, University of Zagreb, Croatia

Tamajo, E., Pavlovski, K., \& Southworth, J. 2011, A\&A, 526, A76

Tapia, M., Costero, R., Echevarria, J., \& Roth, M. 1991, MNRAS, 253, 649

Tomasella, L., Munari, U., Cassisi, S., et al. 2008a, A\&A, 483, 463 (Paper III)

Tomasella, L., Munari, U., Siviero, A., et al. 2008b, A\&A, 480, 465 (Paper II)

Torres, G., Andersen, J., \& Giménez, A. 2010, A\&ARv, 18, 67

Vacca, W. D., Garmany, C. D., \& Shull, J. M. 1996, ApJ, 460, 914

Van Hamme, W. 1993, AJ, 106, 2096

Van Leeuwen, F. 2007, Hipparcos, the new reduction (Springer)

Van Leeuwen, F., \& Fantino, E. 2005, A\&A, 439, 791

Weitenbeck, A. J., Halstead, E. A., \& Carver, A. J. 2008, Acta Astron., 58, 41

Wesselink, A. J. 1941, Annalen van de Sterrewacht te Leiden, 17, 1

Wilson, R. E. 1998, Computing Binary Star Observables, Univ. of Florida Astronomy Dept.

Wilson, R. E., \& Devinney, E. J. 1971, ApJ, 166, 605

Yadav, R. K. S., \& Sagar, R. 2001, MNRAS, 328, 370 